\title{
An Analytic Evolutionary Algorithm To Compute Higher-Order Solutions For Model Equation
}

\section{Bang-Qing Li}

Beijing Technology and Business University

Yu-Lan Ma ( $\sim$ mayl@th.btbu.edu.cn )

Beijing Technology and Business University https://orcid.org/0000-0003-3205-1729

\section{Research Article}

Keywords: evolutionary computing, analytic evolutionary algorithm, Darboux operator, model equation, higher-order solution

Posted Date: April 26th, 2021

DOl: https://doi.org/10.21203/rs.3.rs-229532/v1

License: (1) This work is licensed under a Creative Commons Attribution 4.0 International License. Read Full License 


\title{
An analytic evolutionary algorithm to compute higher-order solutions for model equation
}

\author{
Bang-Qing $\mathrm{Li}^{\mathrm{a}}$, Yu-Lan Ma*,b \\ ${ }^{a}$ School of Computer, Beijing Technology and Business University, Beijing, 100048, PR China \\ ${ }^{b}$ School of Mathematics and Statistics, Beijing Technology and Business University, Beijing, 100048, PR China
}

\begin{abstract}
In this work, by introducing Darboux operator in evolutionary computing frame, we propose a novel analytic evolutionary algorithm to obtain exact higher-order iteration solutions for model equation. We construct the first-, second- and third-order solutions of a generalized Schrödinger equation by applying this algorithm. The higher-order solutions not only retain the basic features of the lower-order cases, but also become more abundant than the lower-order cases.

Key words: evolutionary computing; analytic evolutionary algorithm; Darboux operator; model equation; higher-order solution
\end{abstract}

\section{Introduction}

In soft computing field, evolutionary computation (EC) is a large class of algorithms inspired by biological evolution. In technical terms, EC is often based on a family of population-based trial and error problem solvers with a metaheuristic or stochastic optimization character [1]. In general, as EC is performed, an initial set of solutions can be generated and iteratively updated. Each new generation can be produced by stochastically removing less desired solutions, and introducing small random changes. As a result, the solutions obtained are numeric and approximate [2].

As we know, the analytic solutions play a fundamental role in a variety of application from science to engineering and technology because they are more robust and reliable than the corresponding approximate solutions [3, 4]. Especially, as the number of iterations increases, the availability of the approximate solutions is greatly reduced due to the accumulation of errors. However, obtaining the analytic solutions is very difficult and challenging for most models. Fortunately, with the great development in computer hardware and symbolic computation technique, the analytical solutions of some models, which can only be solved by numerical method before, have also been obtained by a lot of new methods. How to generate the analytic solutions by EC? Darboux transformation (DT) theory, a classical mathematical tool, may provide us a new insight.

DT is named by Jean Gaston Darboux who established Darboux theorem as the solution of the Pfaff problem [5]. Darboux theorem is a powerful mathematical tool and mainly applied in differential

\footnotetext{
* Corresponding author

Email addresses: libq@th.btbu.edu.cn (Bang-Qing Li), mayl@th.btbu.edu.cn (Yu-Lan Ma)
} 
geometry. DT is a simultaneous mapping between solutions and coefficients of a pair of equations (or systems). Gu et. al. extended this theorem to construct the analytic soliton solutions of the well-known Yang-Mills-Higgs equations [6, 7]. Since then, many important works have achieved based on the DT technique [8-13].

Inspired by the core idea implied in DT technique and EC, our objective in this work is to combine both and build a new EC algorithm, namely, analytic evolutionary algorithm (AEA). We extract a few critical elements in DT: initial seed solution, DT operator and iteration, which exactly meet the essential features of EC: initial solution set, change operator and iteratively update. However, there is an obvious difference between them, which is that the change operator during each AEA iterating process is definite. It results the analytic solution will be generated. Of course, the higher-order solutions hold on basic features of lower-order cases.

In the next section, we will introduce AEA. Then, we give an example by applying AEA to solve the higher-order analytic solutions for a generalized Schrödinger equation in Section 3. A few conclusions are given in the final section.

\section{A depict for the analytical evolution algorithm}

Step 1: To determine the Lax equations of the model equation.

Step 2: To generate the first-, second- and multiple-order operator.

Step 3: To choose the initial seed solution of the model equation.

Step 4: To generate the first-order solution by mixing the seed solution and the first-order operator.

Step 5: To generate the second-order solution by mixing the first-order solution and the second-order operator.

Step 6: To perform the similar way in the above steps 4-5, the higher-order (or Nth-order) solutions can be generated.

The algorithm diagram is illustrated in Fig. 1.

Remark: For the above steps, Steps 1 and 3 are usually achieved by using mathematical derivation according to the particular model equation. The other steps can be automatically calculated by symbolic computation software, such as Maple and Mathematics.

\section{An application example of AEA}

In this section, we take a generalized nonlinear Schrödinger equation in nonlinear optics as example to illustrate the application of AEA. The model equation reads as [14]

$$
i u_{x}+i \beta_{1}(x) u_{t}+\beta_{2}(x) u_{t t}+\gamma(x)|u|^{2} u=0,
$$

where $u=u(x, t)$ represents complex pulse wave along optical fibers, $i=\sqrt{-1}$ is imaginary unit, $\beta_{1}(x)$ and $\beta_{2}(x)$ are two types of group velocity dispersion coefficients corresponding to the first and second dispersion terms, respectively; $\gamma(x)$ is a variable nonlinearity coefficient. Eq. (1) can be used to describe 


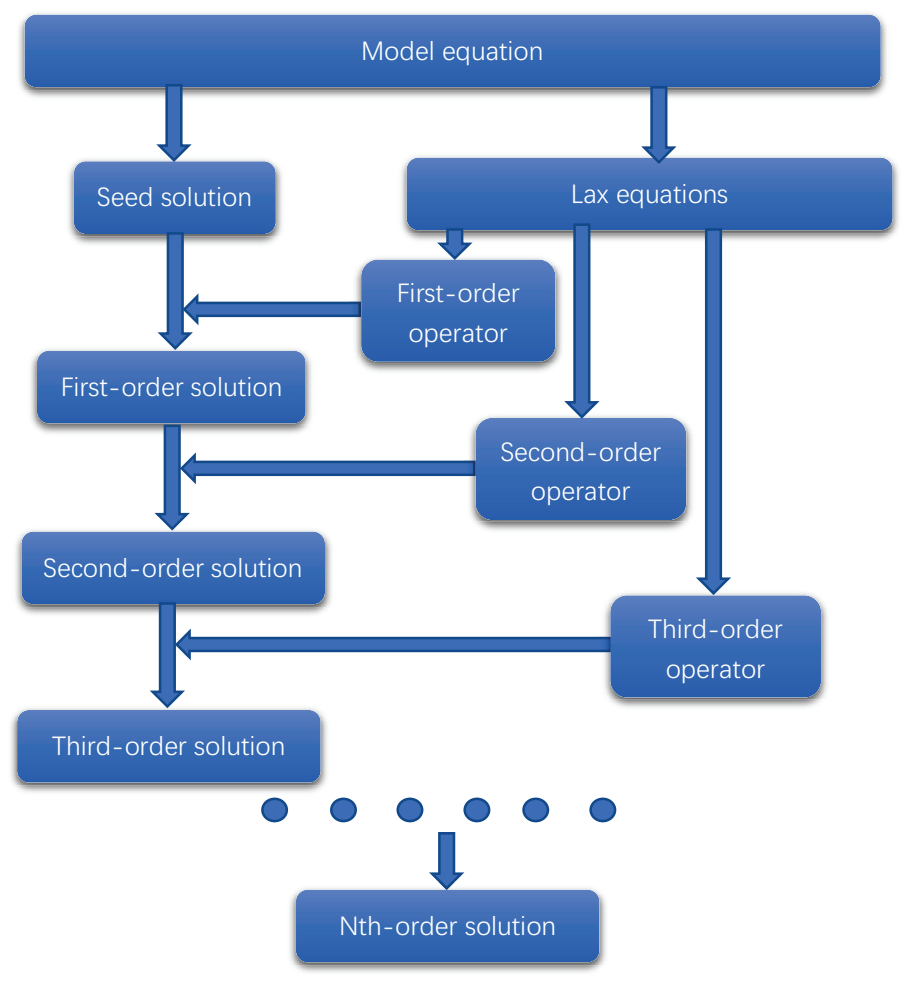

Fig. 1: The algorithm diagram of AEA

the optical pulse propagation in nonlinear optics and studied by scholars. In Ref. [15], a type of oscillating solitons was obtained by the bilinear method for Eq. (1). In Ref. [16], the explicit rogue wave solutions were constructed via similarity transformation, and the first- and second-order rogue waves were excited. Novel phase shift, amplification, oscillation and attenuation of optical solitons were first studied in details by Hirota bilinear method to Eq. (1) in Ref. [17]. According to the two soliton solutions obtained, $\beta_{1}(x)$ and $\gamma(x)$ with different function forms are taken to explore the characteristics of solitons. The effect of the phase shift on the soliton interaction was observed.

\subsection{Lax equations and Darboux operators to Eq. (1)}

Firstly, Eq. (1) has the following integral condition [14]

$$
\gamma(x)=2 \beta_{2}(x) .
$$

Thus, the Lax equations with the integral condition (2) can be expressed as

$$
\Psi_{t}=U \Psi, \Psi_{x}=V \Psi
$$

where

$$
\begin{aligned}
& U=i \lambda \sigma_{1}+i Q, \\
& V=i 2 \lambda^{2} \beta_{2}(x) \sigma_{1}+i \lambda\left(-\beta_{1}(x) \sigma_{1}+2 \beta_{2}(x) Q\right)-i \beta_{1}(x) Q+\beta_{2}(x) \sigma_{1}\left(Q_{t}-i Q^{2}\right),
\end{aligned}
$$


with

$$
\begin{aligned}
& \sigma_{1}=\left(\begin{array}{cc}
1 & 0 \\
0 & -1
\end{array}\right), \\
& Q=\left(\begin{array}{ll}
0 & u^{*} \\
u & 0
\end{array}\right),
\end{aligned}
$$

in which, the asterisk $*$ on the upper-right corner of $u$ represents the conjugate of $u$, and this denotation is also applicable to other identifiers in this article.

Now, we define the DT as

$$
\begin{aligned}
& \Psi[1]=T[1] \Psi=\left(\lambda I-M^{[1]}\right) \Psi \\
& \Psi_{t}[1]=U^{[1]} \Psi[1], \Psi_{x}[1]=V^{[1]} \Psi[1]
\end{aligned}
$$

and $T[1]$ satisfies

$$
\begin{aligned}
& T_{t}[1]+T[1] U^{[0]}=U^{[1]} T[1], \\
& T_{x}[1]+T[1] V^{[0]}=V^{[1]} T[1],
\end{aligned}
$$

where

$$
\begin{aligned}
M^{[1]} & =\left(\begin{array}{rr}
m_{11}^{[1]} & m_{12}^{[1]} \\
m_{21}^{[1]} & m_{22}^{[1]}
\end{array}\right), I=\left(\begin{array}{ll}
1 & 0 \\
0 & 1
\end{array}\right), \\
U^{[k]} & =i \lambda \sigma_{1}+i Q^{[k]} \\
V^{[k]} & =i 2 \lambda^{2} \beta_{2}(x) \sigma_{1}+i \lambda\left(-\beta_{1}(x) \sigma_{1}+2 \beta_{2}(x) Q^{[k]}\right)-i \beta_{1}(x) Q^{[k]} \\
& +\beta_{2}(x) \sigma_{1}\left(Q_{t}^{[k]}-i\left(Q^{[k]}\right)^{2}\right),
\end{aligned}
$$

with

$$
Q^{[k]}=\left(\begin{array}{cc}
0 & u^{[k]^{*}} \\
u^{[k]} & 0
\end{array}\right), k=0,1 .
$$

Substituting $T[1]=\lambda I-M^{[1]}, T_{t}^{[1]}=-M_{t}^{[1]}$ and (10)-(12) into (8), it will yield

$$
-M_{t}^{[1]}+\left(\lambda I-M^{[1]}\right)\left(i \lambda \sigma_{1}+i Q^{[0]}\right)=\left(i \lambda \sigma_{1}+i Q^{[1]}\right)\left(\lambda I-M^{[1]}\right) .
$$

Collecting the terms with the same power with respect to $\lambda^{k}$, and letting their coefficients be zero, it leads a set of algebraic equations as follows

$$
\begin{aligned}
& \lambda^{2}: i I \sigma_{1}-i \sigma_{1} I=0, \\
& \lambda: i Q^{[1]}-i Q^{[0]}+i M^{[1]} \sigma_{1}-i \sigma_{1} M^{[1]}=0, \\
& \lambda^{0}:-M_{t}^{[1]}-i M^{[1]} Q^{[0]}+i Q^{[1]} M^{[1]}=0 .
\end{aligned}
$$


From the equations (15), we get

$$
Q^{[1]}=Q^{[0]}+\left(\sigma_{1} M^{[1]}-M^{[1]} \sigma_{1}\right) .
$$

Therefore, it follows

$$
u^{[1]}=u^{[0]}-2 m_{21}^{[1]},
$$

where $m_{21}^{[1]}$ is the first-order DT operator.

Next, we derive $M^{[1]}$. Supposing

$$
M^{[1]}=L_{1} \Lambda L_{1}^{-1},
$$

and chooseing

$$
L_{1}=\left(\begin{array}{ll}
\psi_{11} & \psi_{12} \\
\psi_{21} & \psi_{22}
\end{array}\right), \Lambda=\left(\begin{array}{cc}
\lambda_{1} & 0 \\
0 & \lambda_{2}
\end{array}\right), L_{1}^{-1}=\frac{1}{\Delta_{1}}\left(\begin{array}{cc}
\psi_{22} & -\psi_{12} \\
-\psi_{21} & \psi_{11}
\end{array}\right)
$$

then substituting (20) into (19), it leads

$$
M^{[1]}=\left(\begin{array}{cc}
m_{11}^{[1]} & m_{12}^{[1]} \\
m_{21}^{[1]} & m_{22}^{[1]}
\end{array}\right)=\frac{1}{\Delta_{1}}\left(\begin{array}{cc}
\lambda_{1} \psi_{11} \psi_{22}-\lambda_{2} \psi_{12} \psi_{21} & \left(\lambda_{2}-\lambda_{1}\right) \psi_{11} \psi_{12} \\
\left(\lambda_{1}-\lambda_{2}\right) \psi_{21} \psi_{22} & \lambda_{2} \psi_{11} \psi_{22}-\lambda_{1} \psi_{12} \psi_{21}
\end{array}\right)
$$

where

$$
\Delta_{1}=\psi_{11} \psi_{22}-\psi_{12} \psi_{21}
$$

Therefore, we have the following proposition.

Proposition. Suppose that $\Psi=\left(\psi_{1 j}, \psi_{2 j}\right)^{T}, j=1,2$, is the solution of Eq. (4) under the same potential $u$ with respect to $\lambda=\lambda_{j}$, and let $L_{1}=\left(\begin{array}{cc}\psi_{11} & \psi_{12} \\ \psi_{21} & \psi_{22}\end{array}\right)$, and $M^{[1]}=L_{1} \Lambda L_{1}^{-1}, \Lambda=$

$$
\begin{gathered}
\left(\begin{array}{cc}
\lambda_{1} & 0 \\
0 & \lambda_{2}
\end{array}\right), T[1]=\lambda I-M^{[1]}, \text { then } \Psi^{[1]}=T[1] \Psi \text { satisfies } \\
\Psi_{t}^{[1]}=U^{[1]} \Psi^{[1]}, \Psi_{x}^{[1]}=V^{[1]} \Psi^{[1]},
\end{gathered}
$$

where the matrices $U^{[1]}$ and $V^{[1]}$ have the same forms as the matrices $U$ and $V$; except that the potential $u$ us substituted by the new potential $u^{[1]}$. Moreover,

$$
u^{[1]}=u^{[0]}-2\left(\lambda_{1}-\lambda_{2}\right) \frac{\psi_{21} \psi_{22}}{\psi_{11} \psi_{22}-\psi_{21} \psi_{12}},
$$

is the first-order solution of Eq. (1). And the AEA process can be worked successively as follows

$$
\left(u^{[0]}, \Psi\right) \rightarrow\left(u^{[1]}, \Psi^{[1]}\right) \rightarrow\left(u^{[2]}, \Psi^{[2]}\right) \rightarrow\left(u^{[3]}, \Psi^{[3]}\right) \rightarrow \cdots
$$

where $\Psi, \Psi^{[1]}, \Psi^{[2]}, \Psi^{[3]}, \cdots$ include iteration operators for each order. 
3.2. analytic breather solutions for Eq. (1)

The choice to the initial seed solution $u^{[0]}$ is significant for the further iteration solutions. Here, we first assume $u^{[0]}$ has the form as

$$
u^{[0]}=e^{i(f+g)}
$$

where

$$
f=\int\left[\gamma(x)-a\left(\beta_{1}(x)+a \beta_{2}(x)\right)\right] d x, g=a t, a \in R .
$$

By substituting (25) into Eq. (1), and considering the condition (2), we can derive that $f(x)$ and $g(t)$ involved in $u^{[0]}$ satisfy

$$
f=\left(2-a^{2}\right) \int \beta_{2}(x) d x-a \int \beta_{1}(x) d x, g=a t, a \in R .
$$

This results

$$
u^{[0]}=e^{i\left[\left(2-a^{2}\right) \int \beta_{2}(x) d x-a \int \beta_{1}(x) d x+a t\right]} .
$$

Through computation, we are able to obtain a set of the solutions of the Lax equations (3) with (25) corresponding to the parameter $\lambda_{1}$ as

$$
\Phi_{1}=\left(\begin{array}{c}
\phi_{11} \\
\phi_{21}
\end{array}\right)=\left(\begin{array}{c}
\left(c_{1} e^{\xi}+e^{-\xi}\right) e^{-\frac{i}{2}(f+g)} \\
\left(-c_{1} e^{-\xi}+e^{\xi}\right) e^{\frac{i}{2}(f+g)}
\end{array}\right)
$$

and another set of the solutions of the Lax equations (3) with (25) corresponding to the parameter $\lambda_{2}=\lambda_{1}^{*}$ as

$$
\Phi_{2}=\left(\begin{array}{c}
\phi_{12} \\
\phi_{22}
\end{array}\right)=\left(\begin{array}{c}
-\left(e^{\xi^{*}}-c_{1}^{*} e^{-\xi^{*}}\right) e^{-\frac{i}{2}(f+g)} \\
\left(c_{1}^{*} e^{\xi^{*}}+e^{-\xi^{*}}\right) e^{\frac{i}{2}(f+g)}
\end{array}\right),
$$

where

$$
\begin{aligned}
& \xi=\frac{i}{2} \sqrt{a^{2}+4\left(\lambda_{1}^{2}+a \lambda_{1}+1\right)}\left(t+\left(2 \lambda_{1}-a\right) \int \beta_{2}(x) d x-\int \beta_{1}(x) d x\right), \\
& c_{1}=\frac{1}{2}\left(\sqrt{a^{2}+4\left(\lambda_{1}^{2}+a \lambda_{1}+1\right)}+2 \lambda_{1}+a\right) .
\end{aligned}
$$

By the same way, we get a set of the solutions of the Lax equations (3) with (25) corresponding to the parameter $\lambda_{3}$ as

$$
\Phi_{3}=\left(\begin{array}{c}
\phi_{13} \\
\phi_{23}
\end{array}\right)=\left(\begin{array}{c}
\left(c_{3} e^{\xi_{3}}+e^{-\xi_{3}}\right) e^{-\frac{i}{2}(f+g)} \\
\left(-c_{3} e^{-\xi_{3}}+e^{\xi_{3}}\right) e^{\frac{i}{2}(f+g)}
\end{array}\right),
$$

and another set of the solutions of the Lax equations (3) with (25) corresponding to the parameter $\lambda_{4}=\lambda_{3}^{*}$ as

$$
\Phi_{4}=\left(\begin{array}{c}
\phi_{14} \\
\phi_{24}
\end{array}\right)=\left(\begin{array}{c}
-\left(e^{\xi_{3}^{*}}-c_{3}^{*} e^{-\xi_{3}^{*}}\right) e^{-\frac{i}{2}(f+g)} \\
\left(c_{3}^{*} e^{\xi_{3}^{*}}+e^{-\xi_{3}^{*}}\right) e^{\frac{i}{2}(f+g)}
\end{array}\right),
$$


where

$$
\begin{aligned}
& \xi_{3}=\frac{i}{2} \sqrt{a^{2}+4\left(\lambda_{3}^{2}+a \lambda_{3}+1\right)}\left(t+\left(2 \lambda_{3}-a\right) \int \beta_{2}(x) d x-\int \beta_{1}(x) d x\right), \\
& c_{3}=\frac{1}{2}\left(\sqrt{a^{2}+4\left(\lambda_{3}^{2}+a \lambda_{3}+1\right)}+2 \lambda_{3}+a\right) .
\end{aligned}
$$

Further, we can acquire a set of the solutions of the Lax equations (3) with (25) corresponding to the parameter $\lambda_{5}$ as

$$
\Phi_{5}=\left(\begin{array}{c}
\phi_{15} \\
\phi_{25}
\end{array}\right)=\left(\begin{array}{c}
\left(c_{5} e^{\xi_{5}}+e^{-\xi_{5}}\right) e^{-\frac{i}{2}(f+g)} \\
\left(-c_{5} e^{-\xi_{5}}+e^{\xi_{5}}\right) e^{\frac{i}{2}(f+g)}
\end{array}\right)
$$

and another set of the solutions of the Lax equations (3) with (25) corresponding to the parameter $\lambda_{6}=\lambda_{5}^{*}$ as

$$
\Phi_{6}=\left(\begin{array}{c}
\phi_{16} \\
\phi_{26}
\end{array}\right)=\left(\begin{array}{c}
-\left(e^{\xi_{5}^{*}}-c_{5}^{*} e^{-\xi_{5}^{*}}\right) e^{-\frac{i}{2}(f+g)} \\
\left(c_{5}^{*} e^{\xi_{5}^{*}}+e^{-\xi_{5}^{*}}\right) e^{\frac{i}{2}(f+g)}
\end{array}\right),
$$

where

$$
\begin{aligned}
\xi_{5} & =\frac{i}{2} \sqrt{a^{2}+4\left(\lambda_{5}^{2}+a \lambda_{5}+1\right)}\left(t+\left(2 \lambda_{5}-a\right) \int \beta_{2}(x) d x-\int \beta_{1}(x) d x\right), \\
c_{5} & =\frac{1}{2}\left(\sqrt{a^{2}+4\left(\lambda_{5}^{2}+a \lambda_{5}+1\right)}+2 \lambda_{5}+a\right) .
\end{aligned}
$$

\subsubsection{First-order breather solution}

Noticing (18)-(21), the first-order breather solution of Eq. (1) can be derived as

$$
u^{[1]}=u^{[0]}-2\left(\lambda_{1}-\lambda_{2}\right) \frac{\phi_{21} \phi_{22}}{\phi_{11} \phi_{22}-\phi_{12} \phi_{21}},
$$

where $u^{[0]}, \phi_{11}, \phi_{12}, \phi_{21}$ and $\phi_{22}$ are given by (28), (29) and (30).

\subsubsection{Second-order breather solution}

We define

$$
\begin{aligned}
& \left(\begin{array}{c}
\phi_{11}[1] \\
\phi_{21}[1]
\end{array}\right) \triangleq\left(\begin{array}{c}
\left(\lambda_{3}-m_{11}^{[1]}\right) \phi_{13}-m_{12}^{[1]} \phi_{23} \\
-m_{21}^{[1]} \phi_{13}+\left(\lambda_{3}-m_{22}^{[1]}\right) \phi_{23}
\end{array}\right), \\
& \left(\begin{array}{c}
\phi_{12}[1] \\
\phi_{22}[1]
\end{array}\right) \triangleq\left(\begin{array}{c}
\left(\lambda_{4}-m_{11}^{[1]}\right) \phi_{14}-m_{12}^{[1]} \phi_{24} \\
-m_{21}^{[1]} \phi_{14}+\left(\lambda_{4}-m_{22}^{[1]}\right) \phi_{24}
\end{array}\right),
\end{aligned}
$$

where

$$
\begin{aligned}
& m_{11}^{[1]}=\frac{\lambda_{1} \phi_{11} \phi_{22}-\lambda_{2} \phi_{12} \phi_{21}}{\Delta_{1}} \\
& m_{12}^{[1]}=\frac{\left(\lambda_{2}-\lambda_{1}\right) \phi_{11} \phi_{12}}{\Delta_{1}} \\
& m_{21}^{[1]}=\frac{\left(\lambda_{1}-\lambda_{2}\right) \phi_{21} \phi_{22}}{\Delta_{1}}
\end{aligned}
$$




$$
m_{22}^{[1]}=\frac{\lambda_{2} \phi_{11} \phi_{22}-\lambda_{1} \phi_{12} \phi_{21}}{\Delta_{1}}
$$

with $\Delta_{1}=\phi_{11} \phi_{22}-\phi_{12} \phi_{21}, \phi_{13}, \phi_{23}, \phi_{14}$ and $\phi_{24}$ are given by (33) and (34).

Further, we can derive

$$
\begin{aligned}
& m_{11}^{[2]}=\frac{\lambda_{3} \phi_{11}[1] \phi_{22}[1]-\lambda_{4} \phi_{12}[1] \phi_{21}[1]}{\Delta_{2}}, \\
& m_{12}^{[2]}=\frac{\left(\lambda_{4}-\lambda_{3}\right) \phi_{11}[1] \phi_{12}[1]}{\Delta_{2}}, \\
& m_{21}^{[2]}=\frac{\left(\lambda_{3}-\lambda_{4}\right) \phi_{21}[1] \phi_{22}[1]}{\Delta_{2}}, \\
& m_{22}^{[2]}=\frac{\lambda_{4} \phi_{11}[1] \phi_{22}[1]-\lambda_{3} \phi_{12}[1] \phi_{21}[1]}{\Delta_{2}} .
\end{aligned}
$$

with $\Delta_{2}=\phi_{11}[1] \phi_{22}[1]-\phi_{12}[1] \phi_{21}[1]$, Thus, the second-order breather solution can be acquired as

$$
u^{[2]}=u^{[1]}-2 m_{21}^{[2]}=u^{[1]}-2 \frac{\left(\lambda_{3}-\lambda_{4}\right) \phi_{21}[1] \phi_{22}[1]}{\phi_{11}[1] \phi_{22}[1]-\phi_{12}[1] \phi_{21}[1]} .
$$

\subsubsection{Third-order breather solution}

Further, we define

$$
\begin{gathered}
\left(\begin{array}{c}
\phi_{11}[2] \\
\phi_{21}[2]
\end{array}\right) \triangleq\left(\begin{array}{cc}
\lambda_{5}-m_{11}^{[2]} & -m_{12}^{[2]} \\
-m_{21}^{[2]} & \lambda_{5}-m_{22}^{[2]}
\end{array}\right)\left(\begin{array}{c}
\left(\lambda_{5}-m_{11}^{[1]}\right) \phi_{15}-m_{12}^{[1]} \phi_{25} \\
\left(\lambda_{5}-m_{22}^{[1]}\right) \phi_{25}-m_{21}^{[1]} \phi_{15}
\end{array}\right), \\
\left(\begin{array}{c}
\phi_{12}[2] \\
\phi_{22}[2]
\end{array}\right) \triangleq\left(\begin{array}{cc}
\lambda_{6}-m_{11}^{[2]} & -m_{12}^{[2]} \\
-m_{21}^{[2]} & \lambda_{6}-m_{22}^{[2]}
\end{array}\right)\left(\begin{array}{l}
\left(\lambda_{6}-m_{11}^{[1]}\right) \phi_{16}-m_{12}^{[1]} \phi_{26} \\
\left(\lambda_{6}-m_{22}^{[1]}\right) \phi_{26}-m_{21}^{[1]} \phi_{16}
\end{array}\right),
\end{gathered}
$$

and

$$
\Delta_{3}=\phi_{11}[2] \phi_{22}[2]-\phi_{12}[2] \phi_{21}[2]
$$

We are able to obtain the third-order breather solution of Eq. (1)

$$
u^{[3]}=u^{[2]}-2 m_{21}^{[3]}=u^{[2]}-2 \frac{\left(\lambda_{5}-\lambda_{6}\right) \phi_{21}[2] \phi_{22}[2]}{\phi_{11}[2] \phi_{22}[2]-\phi_{12}[2] \phi_{21}[2]} .
$$

Following the above precess, the $N$ th solution can be generated $(N>3$ is a positive integer).

In Figs. 2-4, we demonstrate the abundant dynamical properties by visualizing the solutions (41)-(53) under certain settings. It is more interesting that higher-order solutions have more complex and abundant patterns than lower-order cases, while higher-order solutions still retain certain features of lower-order cases.

\section{Conclusions}

In this work, based on the classical DT technique and EC frame, we propose a novel AEA to obtain analytic solutions for application model. We construct the higher-order (from the first-to third-order) solutions of a generalized Schrödinger equation in optics by applying this algorithm. During the solving 
(a)
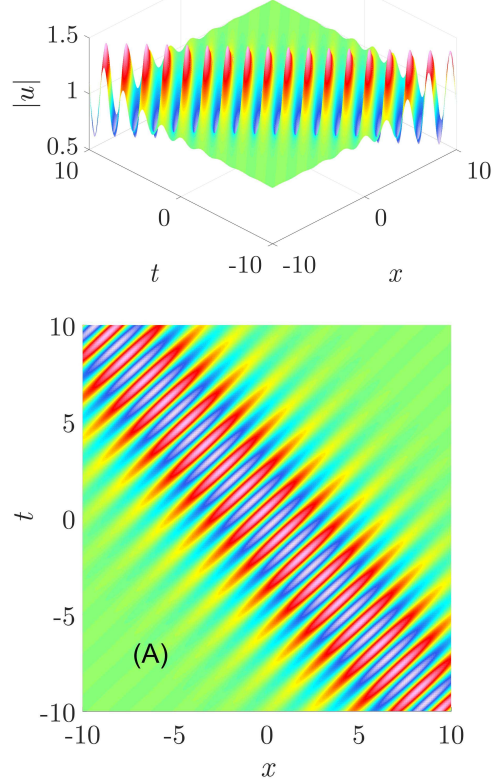

(b)
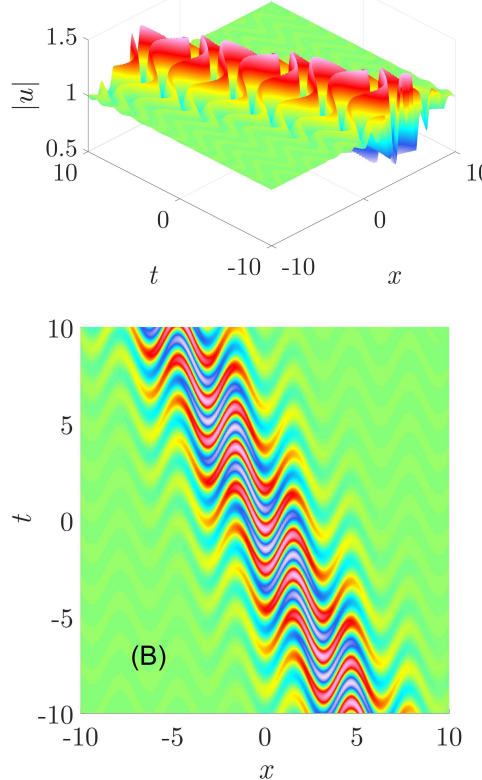

(c)
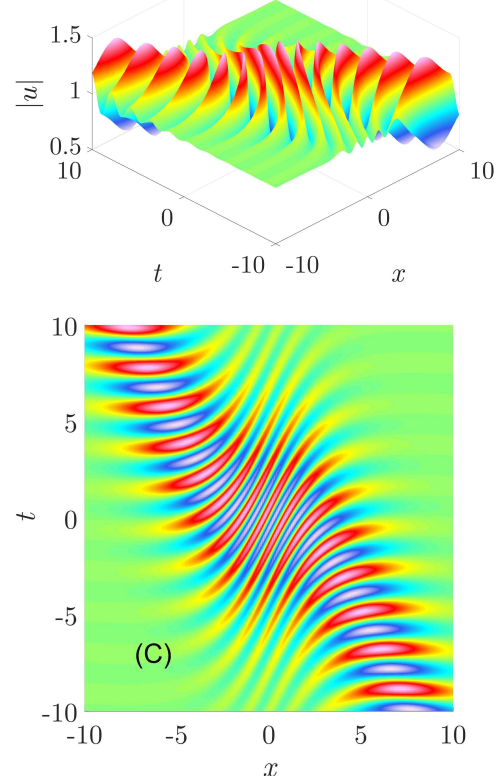

Fig. 2: The first-order breather. The plots are given by the third-order breather solution (41) with the settings: $a=1, \lambda_{1}=$ $0.6+0.2 i, \beta_{2}(x)=0.5$ and different $\beta_{1}(x)$ (a) $\beta_{1}(x)=1$; (b) $\beta_{1}(x)=2 \sin (2 x)$; (c) $\beta_{1}(x)=2 \operatorname{sech}(0.5 x)$. (A)-(C) are the projection views of (a)-(c) on the $x-t$ plane.
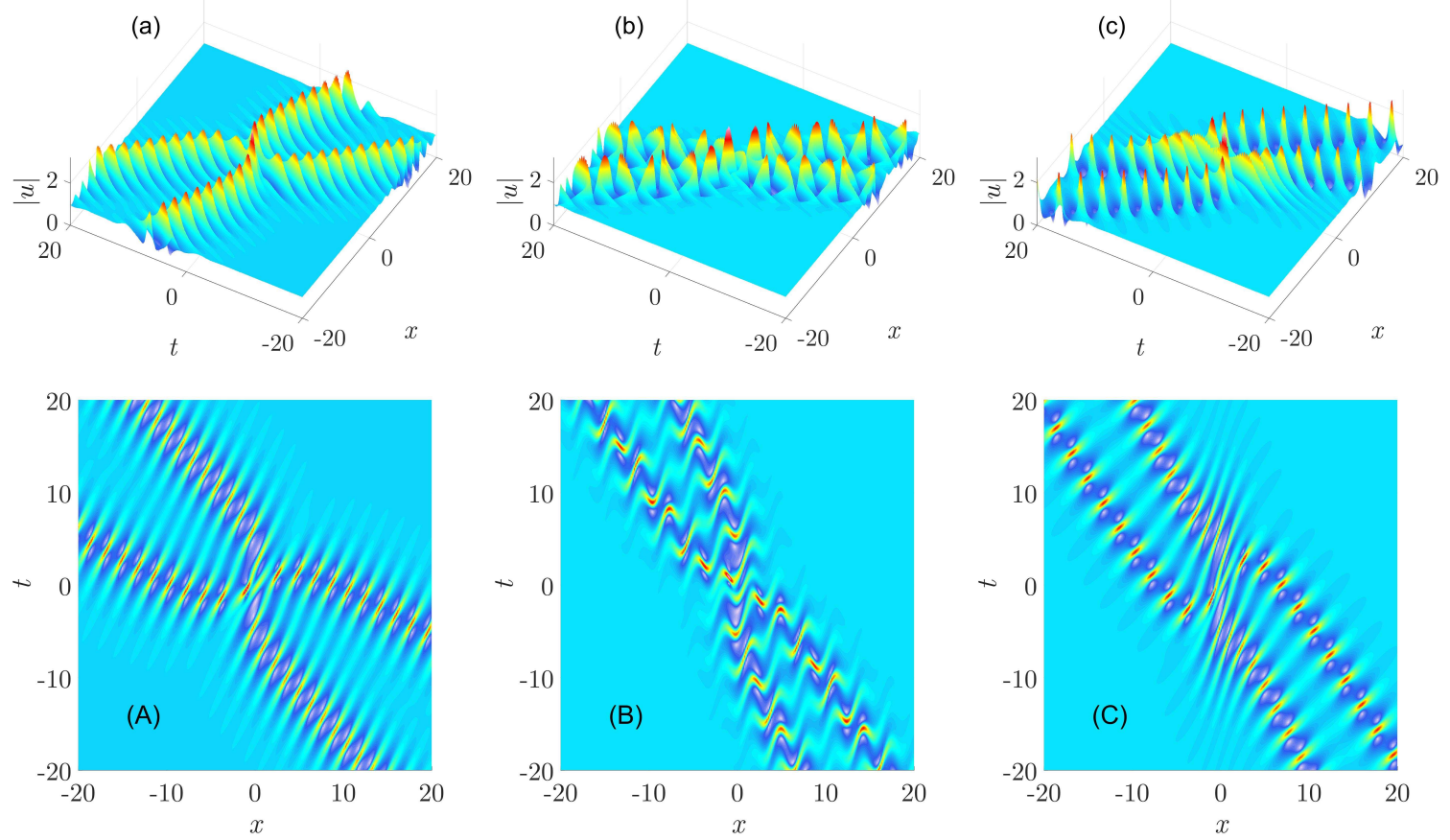

Fig. 3: The second-order breather. The plots are given by the third-order breather solution (41) with the settings: $a=$ $1, \lambda_{1}=-0.3+0.7 i, \lambda_{3}=-0.3+0.8 i, \beta_{2}(x)=0.5$ and different $\beta_{1}(x)$ (a) $\beta_{1}(x)=1 ;$ (b) $\beta_{1}(x)=2 \sin (2 x) ;(\mathrm{c}) \beta_{1}(x)=$ $2 \operatorname{sech}(0.5 x)$. (A)-(C) are the projection views of (a)-(c) on the $x-t$ plane. 
(a)
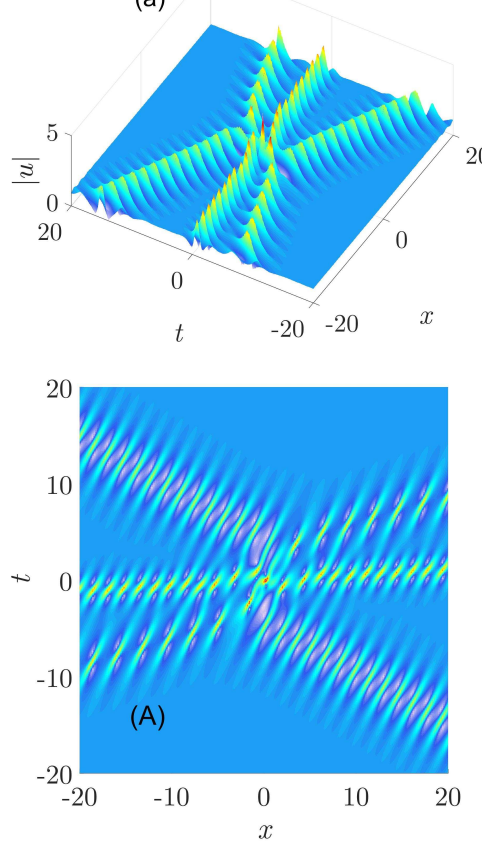

(b)
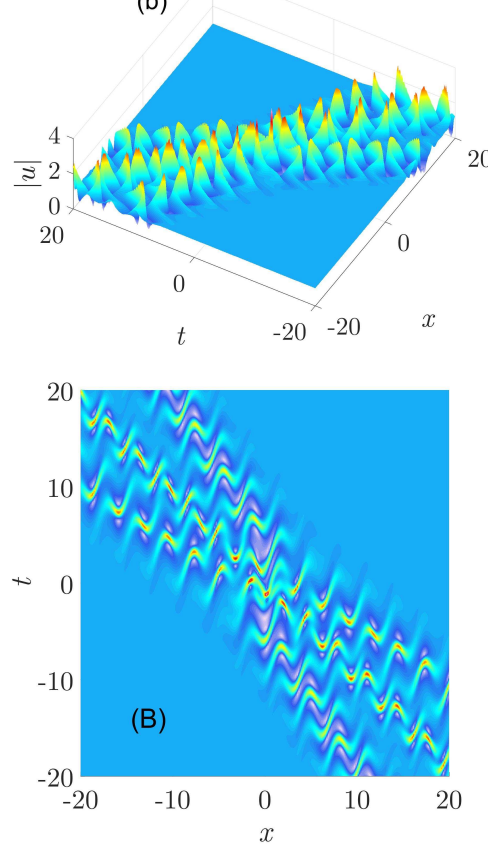

(c)
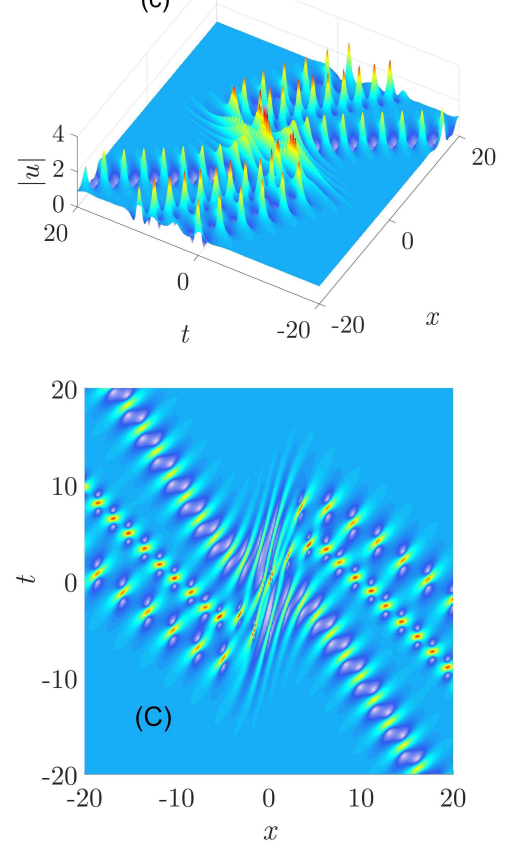

Fig. 4: The third-order breather. The plots are given by the third-order breather solution (53) with the settings: $a=$ $1, \lambda_{1}=0.1+i, \lambda_{3}=-0.2+0.7 i, \lambda_{5}=-0.3+0.9 i, \beta_{2}(x)=0.5$ and different $\beta_{1}(x)$ (a) $\beta_{1}(x)=1 ;$ (b) $\beta_{1}(x)=2 \sin (2 x)$; (c) $\beta_{1}(x)=2 \operatorname{sech}(0.5 x)$. (A)-(C) are the projection views of (a)-(c) on the $x-t$ plane.

process, a series of matrices are derived, which can be generated by computer software. It is fascinating that all the solutions exhibit elegant symmetry.

I think this algorithm extends the boundary of the classic EC algorithm. Consideration of difficulty for seeking for analytic solutions, it is undoubted that AEA has a great application prospective.

\section{Compliance with ethical standards}

\section{Conflict of interest:}

The authors declare that there are no conflicts of interests with publication of this work.

\section{Authorship contributions}

All the authors together conceived and designed the study, then wrote and corrected the manuscript. Ma derived mainly mathematical formulas, while Li programmed the drawing codes to all the solutions. All the authors read and approved the manuscript.

\section{References}

[1] A.E. Eiben, J.E. Smith, Introduction to Evolutionary Computing, Springer, (2015) Second edition. 
[2] A.E. Eiben, J.E. Smith, From evolutionary computation to the evolution of things, Nature 521 (2015) 476.

[3] X. Zhao, J. Peng, J. Liu, X.J. Zhou, Analytic solution of uncertain autoregressive model based on principle of least squares, Soft Comput. 24 (2020) 2721-2726.

[4] H.F. Ismael, H. Bulut, H.M. Baskonus, W-shaped surfaces to the nematic liquid crystals with three nonlinearity laws, Soft Comput. DOI: 10.1007/s00500-020-05459-6

[5] G. Darboux, Lecons sur la théorie général des surfaces, Gauthier-Villars, (1912) Second edition.

[6] C.H. Gu, A.N. Hu, Z.X. Zhou, Darboux Transformations in Integrable Systems: Theory and their Applications to Geometry, Springer, (2004) 2005th edition.

[7] C.H. Gu, Darboux transformation and solitons of Yang-Mills-Higgs equations in R-2,R-1, Sci. China Ser. A-Math. Phys. Astr. 45 (2002) 706.

[8] B.L. Guo, L.M. Li, Q.P. Liu, Nonlinear Schrödinger equation: generalized Darboux transformation and rogue wave solutions, Phys. Rev. E 85 ( 2012) 026607.

[9] Y. Ohta, J.K. Yang, General high-order rogue waves and their dynamics in the nonlinear Schrödinger equation, Proc. R. Soc. A 468 (2012) 1716-1740.

[10] J.S. He, S.W. Xu, K. Porsezian, Rogue wave triggered at a critical frequency of a nonlinear resonant medium, Phys. Rev. E 93 (2016) 062201.

[11] Y.L. Ma, Interaction and energy transition between the breather and rogue wave for a generalized nonlinear Schrödinger system with two higher-order dispersion operators in optical fibers, Nonlinear Dyn. 97 (2019) 95-105.

[12] B.Q. Li, Y.L. Ma, N-order rogue waves and their novel colliding dynamics for a transient stimulated Raman scattering system arising from nonlinear optics, Nonlinear Dyn. 101 (2020) 2449-2461.

[13] B.Q. Li, Y.L. Ma, Extended generalized Darboux transformation to hybrid rogue wave and breather solutions for a nonlinear Schrödinger equation, Appl. Math. Comput. 386 (2020) 125469.

[14] G.P. Agrawal, Nonlinear fiber optics, Academic Press, (2007) Fourth edition.

[15] X.G. Lin, W.J. Liu, M. Lei, Oscillationg solitons in nonlinear optics, Pramana J. Phys. 86 (2016) $575-580$.

[16] B.Q. Li, Y.L. Ma, Rogue waves for the optical fiber system with variable coefficients, Optik 158 (2018) 177-184.

[17] W.T. Yu, Q. Zhou, M. Mirzazadeh, W.J. Liu, A. Biswas, Phase shift, amplification, oscillation and attenuation of solitons in nonlinear optics, J. Adv. Res. 15 (2019) 69-76. 


\section{Figures}

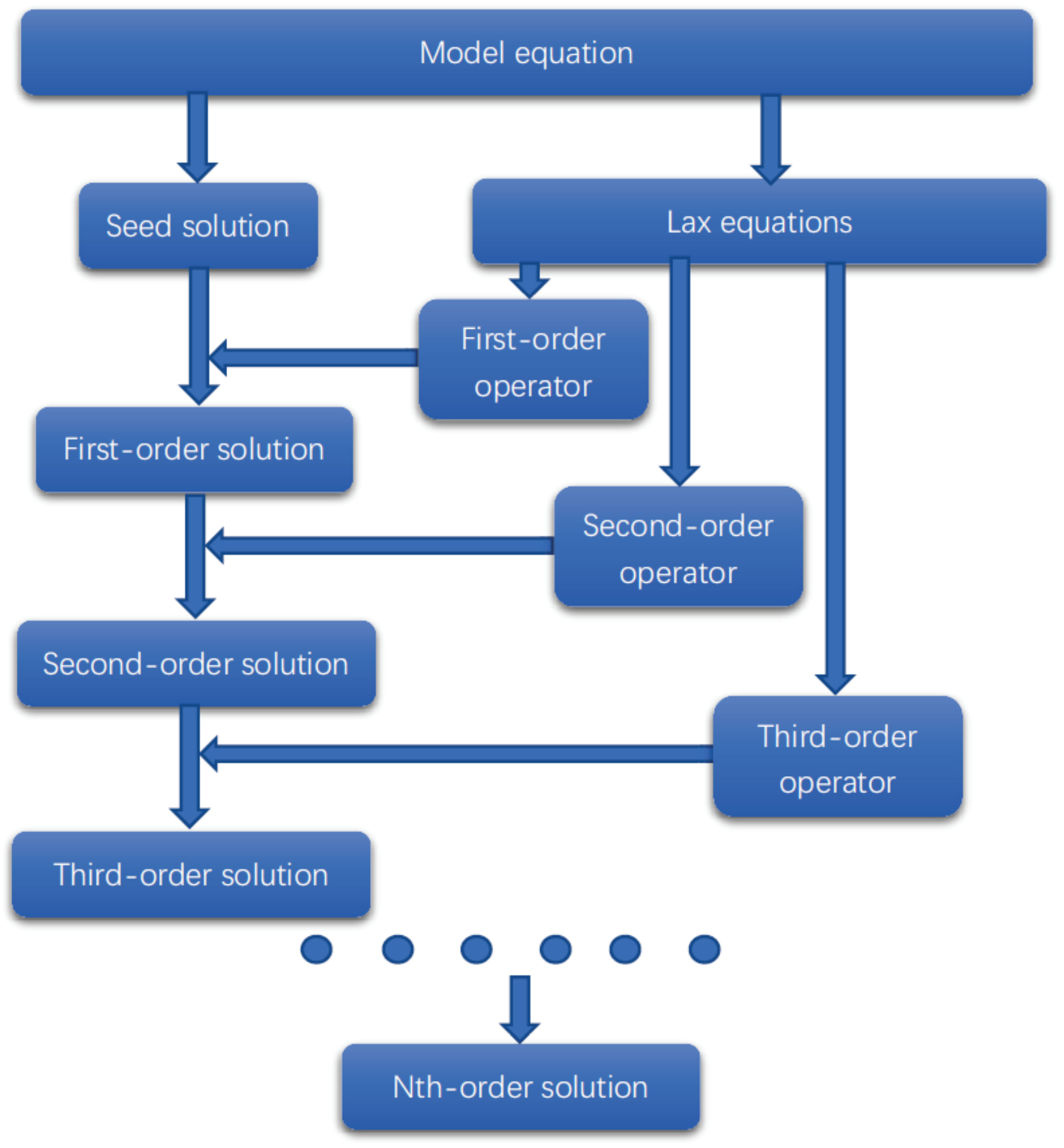

Figure 1

The algorithm diagram of AEA 
(a)
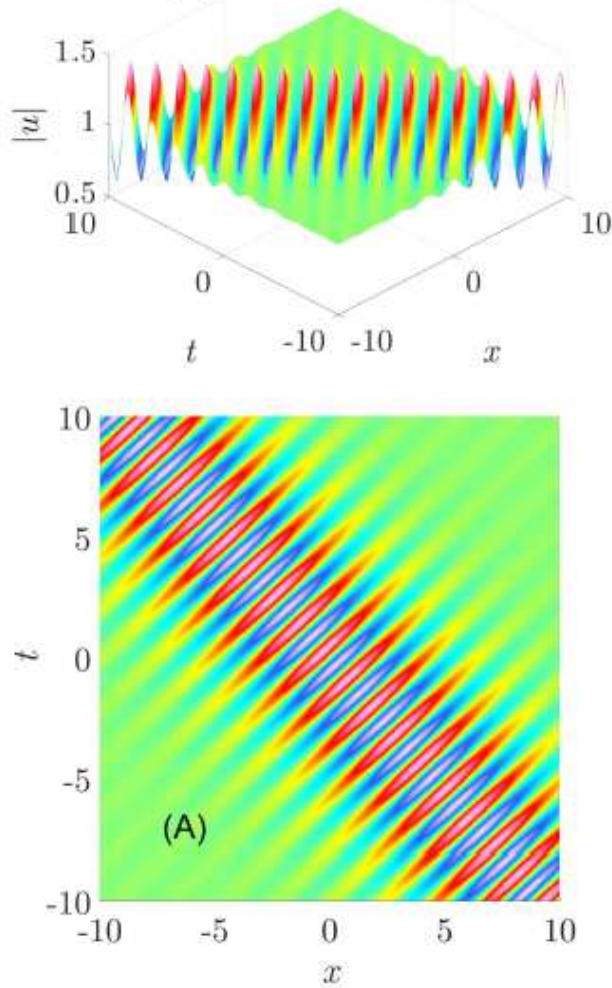

(b)
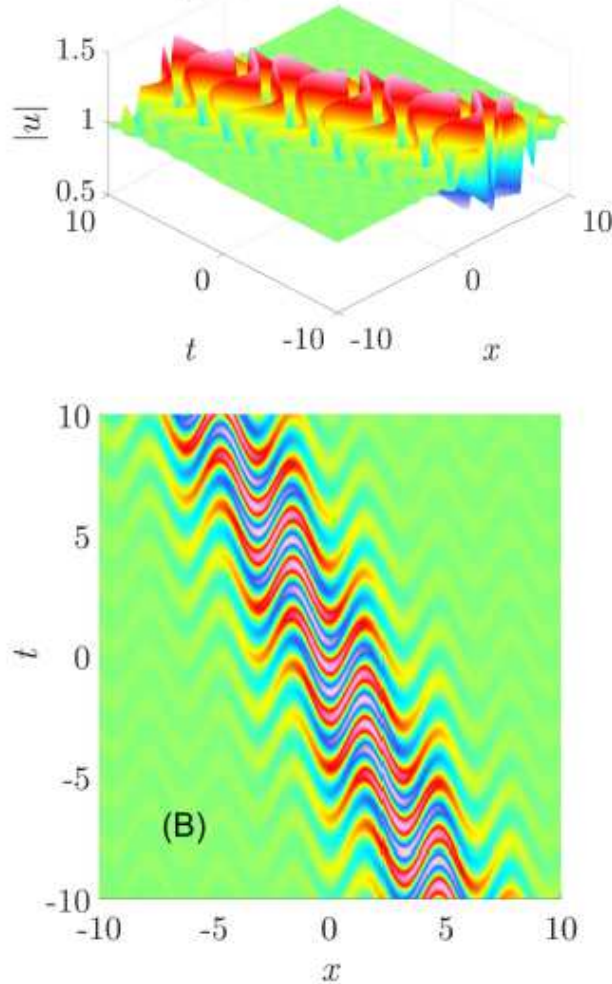

(c)
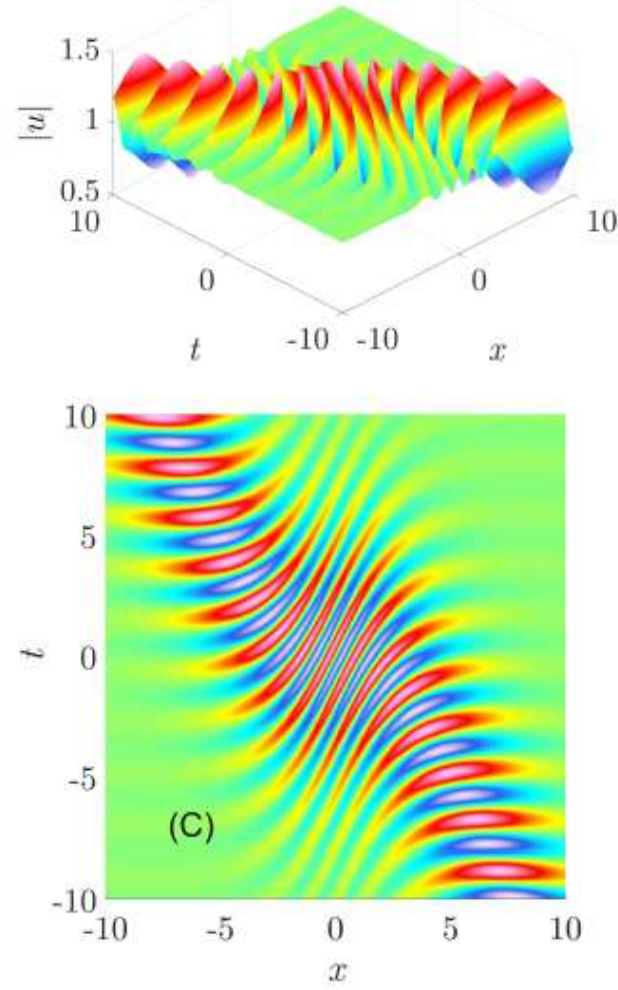

\section{Figure 2}

The first-order breather. The plots are given by the third-order breather solution (41) with the settings: $a=$ $1, \lambda 1=0.6+0.2 \mathrm{i}, \beta 2(\mathrm{x})=0.5$ and different $\beta 1$ (x) (a) $\beta 1(\mathrm{x})=1$; (b) $\beta 1(\mathrm{x})=2 \sin (2 \mathrm{x}) ;(\mathrm{c}) \beta 1(\mathrm{x})=$ $2 \operatorname{sech}(0.5 x) .(A)-(C)$ are the projection views of $(a)-(c)$ on the $x-t$ plane. 
(a)
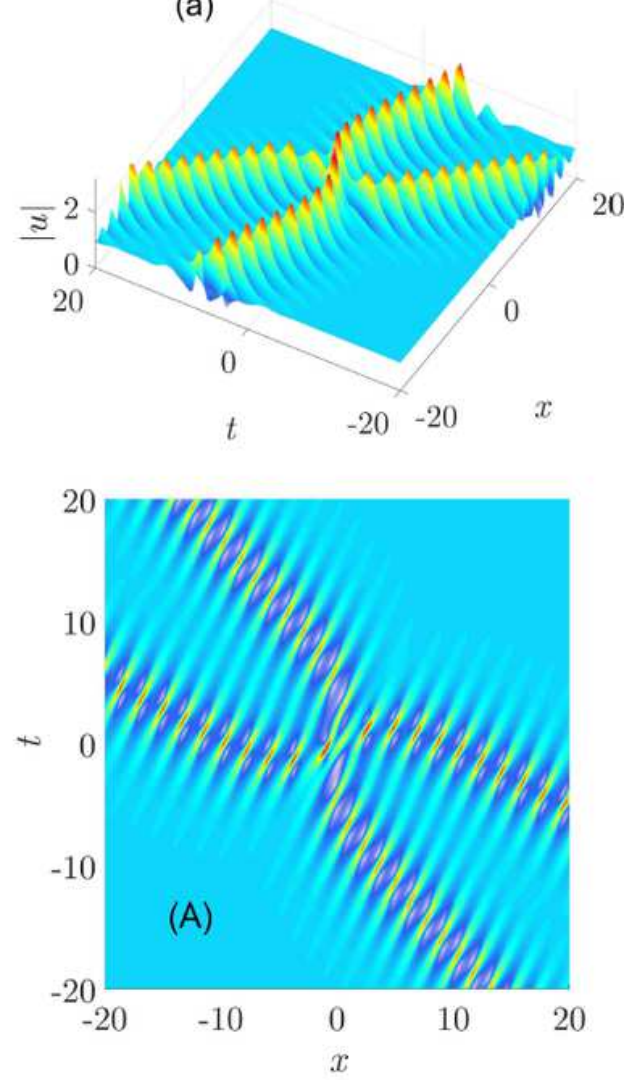

(b)
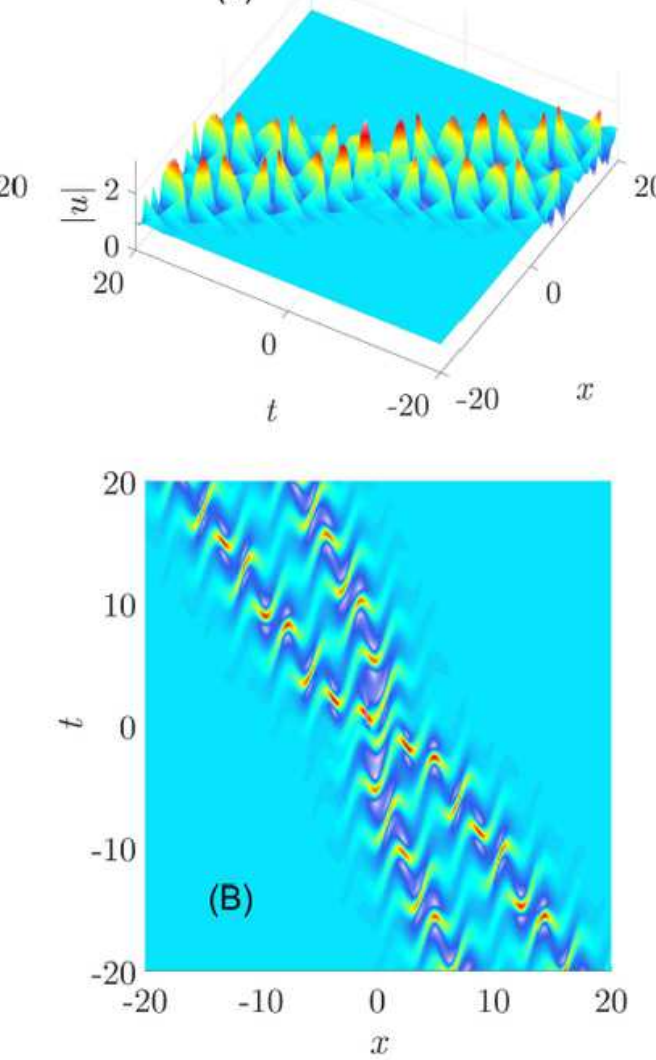

(c)
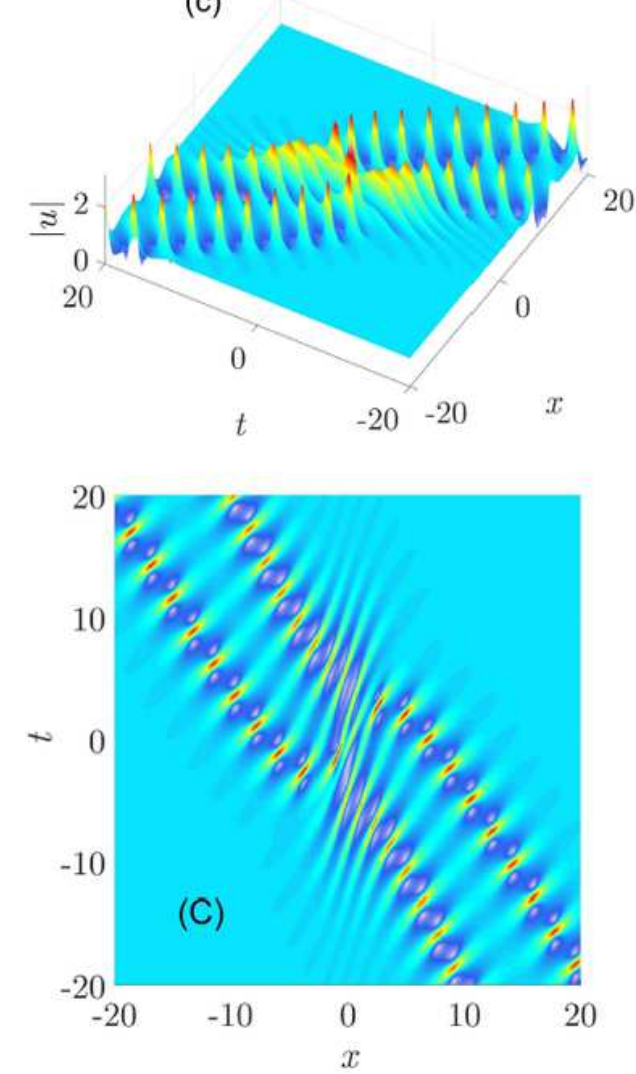

\section{Figure 3}

The second-order breather. The plots are given by the third-order breather solution (41) with the settings: a $=1, \lambda 1=-0.3+0.7 i, \lambda 3=-0.3+0.8 i, \beta 2(x)=0.5$ and different $\beta 1(x)(a) \beta 1(x)=1 ;(b) \beta 1(x)=2 \sin (2 x) ;(c)$ $\beta 1(x)=2 \operatorname{sech}(0.5 x) .(A)-(C)$ are the projection views of $(a)-(c)$ on the $x-t$ plane. 
(a)
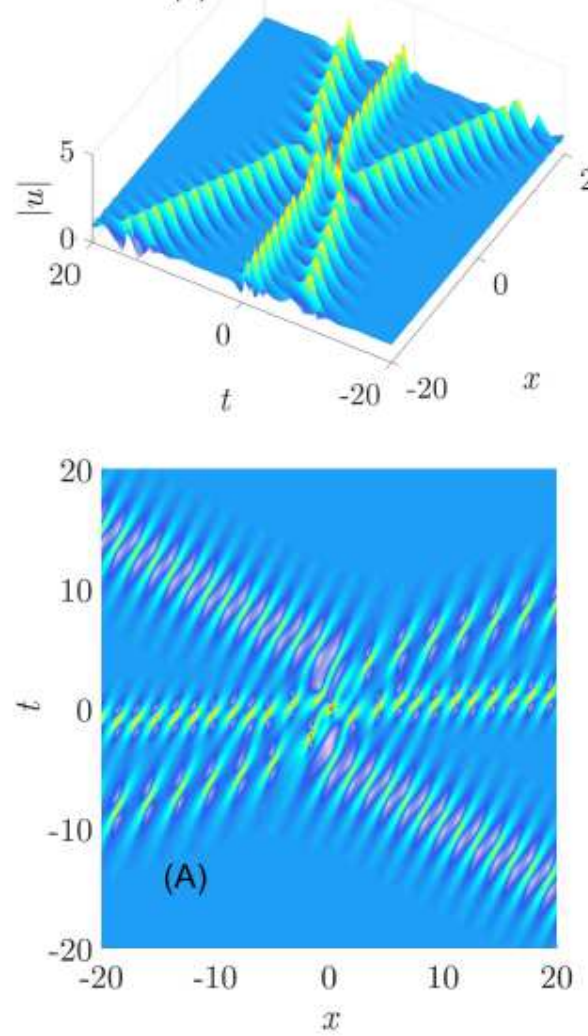

(b)
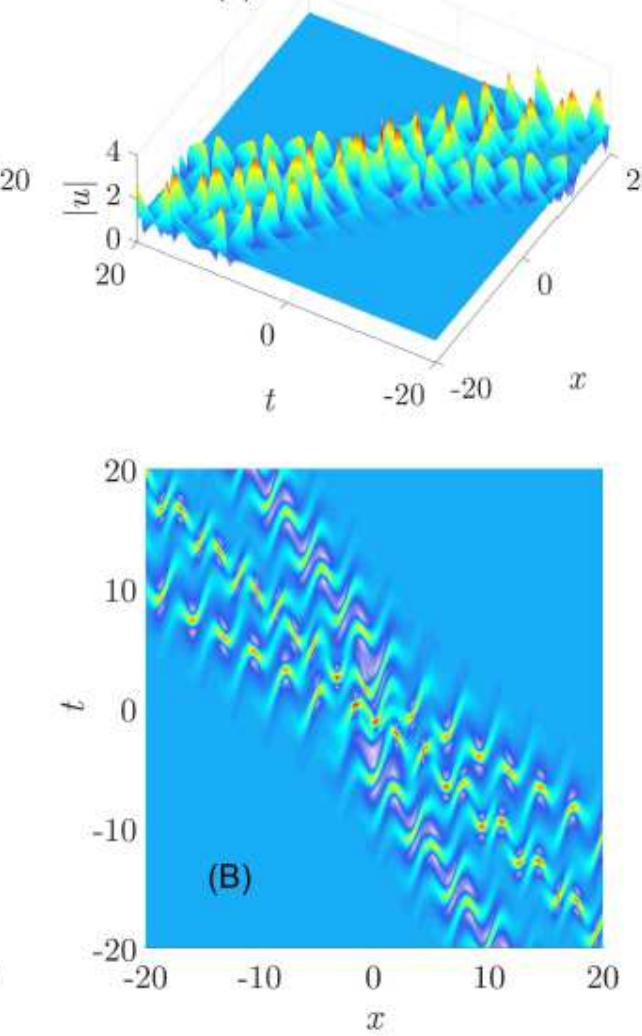

(c)
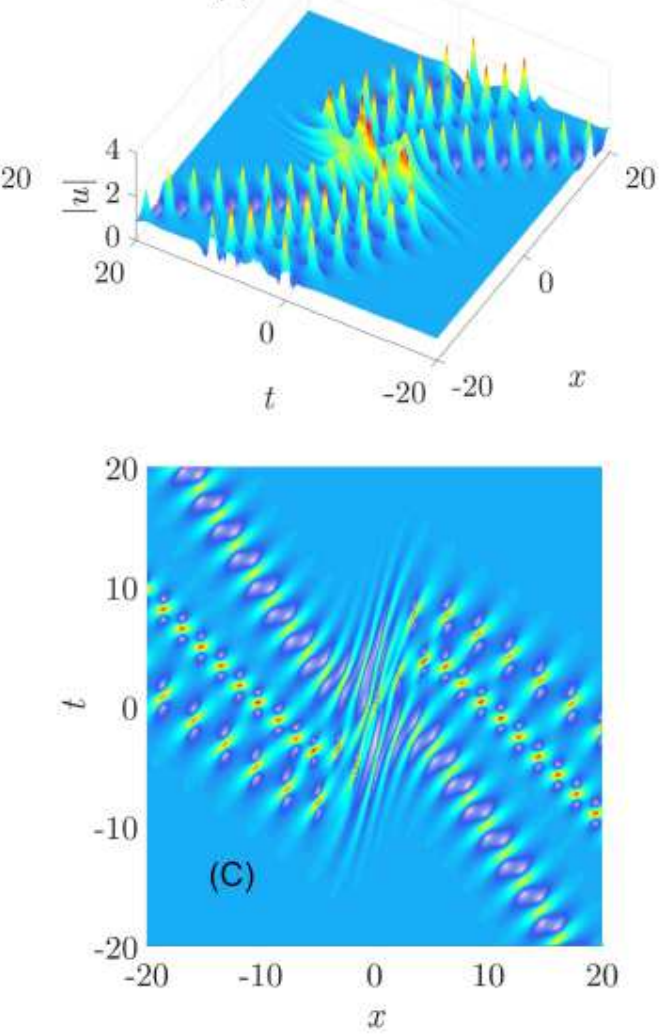

\section{Figure 4}

The third-order breather. The plots are given by the third-order breather solution (53) with the settings: $\mathrm{a}=$ $1, \lambda 1=0.1+i, \lambda 3=-0.2+0.7 i, \lambda 5=-0.3+0.9 i, \beta 2(x)=0.5$ and different $\beta 1(x)(a) \beta 1(x)=1 ;(b) \beta 1(x)=2$ $\sin (2 x) ;(c) \beta 1(x)=\operatorname{sech}(0.5 x)$. (A)-(C) are the projection views of $(a)-(c)$ on the $x-t$ plane. 